\title{
Asymmetric Choroidal Hypopigmentation in a Son and Mother with Waardenburg
}

\section{Syndrome Type I}

Parampal S. Grewal MD FRCSC, Hannah Knight, Michel Michaelides MD, FRCOphth

Moorfields Eye Hospital, London, UK

UCL Institute of Ophthalmology, University College London, London, UK

Correspondence Author:

Professor Michel Michaelides

UCL, Institute of Ophthalmology

11 - 43 Bath Street, London

EC1V 9EL, UK

michel.michaelides@ucl.ac.uk

The authors received no financial support for this research and have no relevant conflict of interest.

Abbreviations: Optical coherence tomography (OCT), Waardenburg syndrome type 1 (WS-I). 
ABSTRACT

\section{Background}

Waardenburg syndrome type I (WS-I) is a rare autosomal-dominant auditory-pigmentary disorder with limited reports in the Ophthalmic literature.

\section{Materials and Methods}

We describe the history, clinical findings and detailed retinal imaging (ultra-widefield fundus images, fundus autofluorescence and optical coherence tomography) from a patient with WS-I.

\section{Case Description}

Our patient had a history of white forelock and congenital hearing loss. Ophthalmic examination demonstrated iris heterochromia and highly asymmetric choroidal hypopigmentation, with generalised fundus hyperautofluorescence. Similarly, the patient's mother demonstrated highly asymmetric fundus hypopigmentation. Genetic testing confirmed a pathogenic PAX3 nonsense variant.

\section{Conclusion}

Our report demonstrates that highly asymmetric choroidal hypopigmentation is within the clinical spectrum of WS-I. 


\section{INTRODUCTION}

Waardenburg Syndrome is a rare autosomal dominant auditory-pigmentary disorder first described by Dutch Ophthalmologist and Geneticist, P.J. Waardenburg in 1951. ${ }^{1}$ Waardenburg syndrome is subdivided into types I to IV based on the specific clinical features. ${ }^{2}$

This report focuses on Waardenburg syndrome type I (WS-I). WS-I is due to disease-causing sequence variants in the gene $P A X 3$ which results in defective neural crest development and melanocyte migration. ${ }^{3}$ It is a heterogenous disorder with incomplete penetrance and variable expressivity. ${ }^{2,4}$ WS-I is characterised by congenital sensorineural hearing loss, pigmentary abnormalities of the hair, skin and eyes and dystopia canthorum without upper limb anomalies. ${ }^{2,5}$ With regards to the ocular manifestations, dystopia canthorum and iris pigmentary abnormalities are commonly described, with fewer publications reporting the posterior segment manifestations. Posterior segment findings include choroidal hypopigmentation which can have a sectoral or diffuse pattern. ${ }^{6,7}$

Herein, we report a novel observation of a mother and son who were found to have unilateral, diffuse choroidal hypopigmentation.

\section{CASE REPORT}

Our patient was a four year old from Brazil who was referred for Ophthalmology assessment by his Audiologist. On presentation, there were no visual concerns. He was noted to have a white forelock and iris heterochromia at birth and failed a hearing screen at 6 months of age. Following identification of hearing loss, he was diagnosed with Waardenburg syndrome and had a cochlear implant. There was some mild delay in his walking, but his growth and development were otherwise normal. They did not report any dermatologic or limb abnormalities. He was systemically well and took no medications. 
There was a family history of his mother having bilateral light irides and white forelock. She had no hearing difficulties. He had no siblings and there was no other family history.

On examination, visual acuity was 0.00 LogMAR in both eyes. There was a low hyperopic refractive error. He had lower puncta located just temporal to the limbus and there was a broad nasal bridge. There was heterochromia with a brown right iris and blue left iris; the iris changes were diffuse without a sectoral pattern. The visual axis was clear with a mild persistent pupillary membrane in the right eye. Fundoscopy demonstrated diffuse hypopigmentation of the left fundus and a relatively normal right fundus with mild nasal hypopigmentation.

Ultra-widefield color and fundus autofluorescence images (Optos Inc, Marlborough, MA, United States) and optical coherence tomography (HRA2, Heidelberg Engineering Ltd, Heidelberg, Germany) from the patient and mother are presented and described in Figures 1 - 3. The left fundus demonstrates diffuse choroidal hypopigmentation and generalized hyperautofluorescence. This is in contrast to the right fundus, with only mild nasal hypopigmentation (Figure 1). Strikingly similar, asymmetric findings are seen in the patient's mother (Figure 3). Optical coherence tomography (OCT) demonstrates normal retinal architecture and infrared reflectance demonstrates readily apparent choroidal vessels (Figure 2). Enhanced depth imaging was unfortunately not obtained. However, to the extent visualised the choroidal thickness appears to be symmetric (based on the position of the choroidal-scleral interface), while the choroidal vasculature is somewhat less distinct.

Genetic testing through next-generation sequencing found our patient was heterozygous for the c.760C > T p.(Gln254*) pathogenic PAX3 nonsense variant. No evidence of a deletion or duplication in the PAX3, MITF or SOX10 genes was found (Rare \& Inherited Disease Genomic Laboratory, London, UK).

\section{DISCUSSION}


Approximately one-third of patients (10/34) with Waardenburg syndrome have been reported to have fundus pigmentary abnormalities. ${ }^{6}$ There are limited detailed reports of the ocular findings. A recent series by Shields et al. reported the ocular manifestations in a series of 7 patients based on clinical examination and multimodal imaging. ${ }^{7}$ In their series, five of seven patients were found to have iris hypopigmentation (8 eyes); this was sectoral in 75\% (6 eyes) and diffuse in 25\% (2 eyes). Similarly, choroidal hypopigmentation was seen in 5 of 7 patients ( 9 eyes), with a sectoral pattern in 6 eyes (67\%) and diffuse in 3 eyes (33\%). Choroidal melanoma has also been reported in a patient with Waardenburg syndrome. Accordingly, the authors suggest patients may benefit from surveillance. $^{8}$

Our report demonstrates that highly asymmetric fundus (and iris) hypopigmentation is possible and within the spectrum of WS-I. To the best of our knowledge, this is the first report with detailed fundus imaging demonstrating such highly asymmetric fundus findings. Of note, in the Shields series of seven patients one patient had unilateral sectoral iris hypopigmentation, this patient did not have any choroidal hypopigmentation. Additionally, one of the patients in whom choroidal hypopigmentation was seen showed unilateral fundus findings along with sectoral iris hypopigmentation. The bilateral cases had a symmetric appearance. ${ }^{7}$ There has been one other report of complete heterochromia with asymmetric fundus pigmentation, though the peripheral fundus findings were not described in this case. ${ }^{9}$

The iris and choroidal changes do not necessarily correspond, as complete heterochromia has been reported with bilateral pigmentary abnormalities. ${ }^{10}$ Our patient had a light iris and hypopigmented choroid on the same side, while his mother had bilateral light irides and only a hypopigmented left fundus. This is plausible embryologically, as choroidal and iris neural crest cells arise from different waves of neural crest cell migration. ${ }^{11}$ 
In terms of other imaging findings, ultra-widefield fluorescein angiography ${ }^{12}$ and OCT angiography ${ }^{13}$ have demonstrated normal retinal vasculature in WS. There have been mixed reports with regards to choroidal thickness, with one case report suggesting choroidal thickening ${ }^{12}$ and others describing decreased choroidal thickness in the areas of hypopigmentation. ${ }^{7,14}$ Our patient appears to have a relatively symmetrical choroidal thickness, albeit less distinct vasculature. The increased fundus autofluorescence is thought to represent the unmasking of scleral hypoautofluorescence. $^{7}$

We identified a heterozygous pathogenic PAX3 nonsense variant (c.760C > T p.(Gln254*)) in our patient. This mutation has been previously reported. Further, it is absent in population databases and would result in a predicted null variant where the loss of function is a known disease mechanism. ${ }^{15,16}$

\section{CONCLUSION}

Our report demonstrates that highly asymmetric choroidal hypopigmentation is within the clinical spectrum of WS-I. 


\section{REFERENCES}

1. Waardenburg PJ. A new syndrome combining developmental anomalies of the eyelids, eyebrows and nose root with pigmentary defects of the iris and head hair and with congenital deafness. Am J Hum Genet. 1951;3(3):195-253.

2. Milunsky JM. Waardenburg Syndrome Type I. GeneReviews. doi:10.7869/djo.2013.14

3. Merchant SN, McKenna MJ, Milunsky A, Baldwin CT, Nadol JB. Otopathology in a case of type I waardenburg's syndrome. Ann Otol Rhinol Laryngol. 2001;110(9):875-882. doi:10.1177/000348940111000913

4. Pardono E, van Bever Y, van den Ende J, et al. Waardenburg syndrome: Clinical differentiation between types I and II. Am J Med Genet. 2003;117A(3):223-235. doi:10.1002/ajmg.a.10193

5. Baldwin CT, Lipsky NR, Hoth CF, Cohen T, Mamuya W, Al BET. Mutations in PAX3 Associated With Waardenburg Syndrome Type I. Hum Mutat. 1994;3:205-211.

6. Delleman JW, Hageman MJ. Ophthalmological findings in 34 patients with Waardenburg syndrome. J Pediatr Ophthalmol Strabismus. 1978;15(6):341-345. doi:10.3928/0191-391319781101-03

7. Shields CL, Nickerson SJ, Al-Dahmash S, Shields JA. Waardenburg syndrome: Iris and choroidal hypopigmentation: Findings on anterior and posterior segment imaging. JAMA Ophthalmol. 2013;131(9):1167-1173. doi:10.1001/jamaophthalmol.2013.4190

8. Itty S, Richter ER, McCannel TA. Choroidal melanoma in a patient with waardenburg syndrome. Retin Cases Br Reports. 2015;9(4):327-329. doi:10.1097/ICB.0000000000000204

9. Bansal Y, Jain P, Goyal G, Singh M, Mishra C. Waardenburg syndrome - A case report. Contact Lens Anterior Eye. 2013;36(1):49-51. doi:10.1016/j.clae.2012.10.083

10. Mullner-Eidenbock A, Moser E, Frisch H, Read AP. Waardenburg syndrome 2 in a Turkish 
family: implications for the importance of the pattern of fundus pigmentation. $\mathrm{Br} J$ Ophthalmol. 2001;85:1384-1393.

11. Levine LM, Brar VS, Goldstein MH, et al. Fundamentals and Principles of Ophthalmology - American Academy of Ophthalmology Basic and Clnical Science Course 2016-2017.

12. Choudhry N, Rao RC. Multi-Modal Ultra-Widefield Imaging Features in Waardenburg Syndrome. Ophthalmic Surg Lasers Imaging Retin. 2015;46(6):670-673.

doi:10.1016/j.physbeh.2017.03.040

13. Hsiao AM, Choudhry N. Swept-Source Optical Coherence Tomography and Optical Coherence Tomography Angiography Findings in Waardenburg Syndrome. Retin Cases Brief Rep. 2015:1. doi:10.1097/icb.0000000000000783

14. Rishi P, Multani P, Prasan VV, Rishi E, Attiku Y. Choroidal thickness in Waardenburg syndrome. GMS Ophthalmol cases. 2019;9. doi:10.3205/oc000111

15. Tassabehji M, Newton VE, Liu XZ, et al. The mutational spectrum in waardenburg syndrome. Hum Mol Genet. 1995;4(11):2131-2137. doi:10.1093/hmg/4.11.2131

16. Barber TD, Barber MC, Cloutier TE, Friedman TB. PAX3 gene structure, alternative splicing and evolution. Gene. 1999;237(2):311-319. doi:10.1016/S0378-1119(99)00339-X 
Figure 1 - Ultra-widefield color and autofluorescence images from the patient.

Ultra-widefield color fundus photographs from the right (A) and left (B) eyes demonstrate mild nasal hypopigmentation in the right and diffuse choroidal hypopigmentation of the left fundus. Corresponding fundus autofluorescence from the right (C) and left (D) eyes demonstrate mild generalized hyperautofluorescence in the left fundus.

Figure 2-Optical coherence tomography images from the patient. The infrared reflectance image of the left eye (B) demonstrates more readily apparent choroidal vessels in comparison to the right eye (A). The corresponding b-scans (C and D) demonstrate normal macular architecture.

Figure 3 - Ultra-widefield color and autofluorescence images from the patient's mother. Ultra-widefield color photographs from the right (A) and left (B) eyes and corresponding fundus autofluorescence ( $\mathrm{C}$ and $\mathrm{D})$ from the mother demonstrate a similar pattern to the son. There is diffuse left choroidal hypopigmentation, with mild generalized hyperautofluorescence. Right eye imaging is normal. 


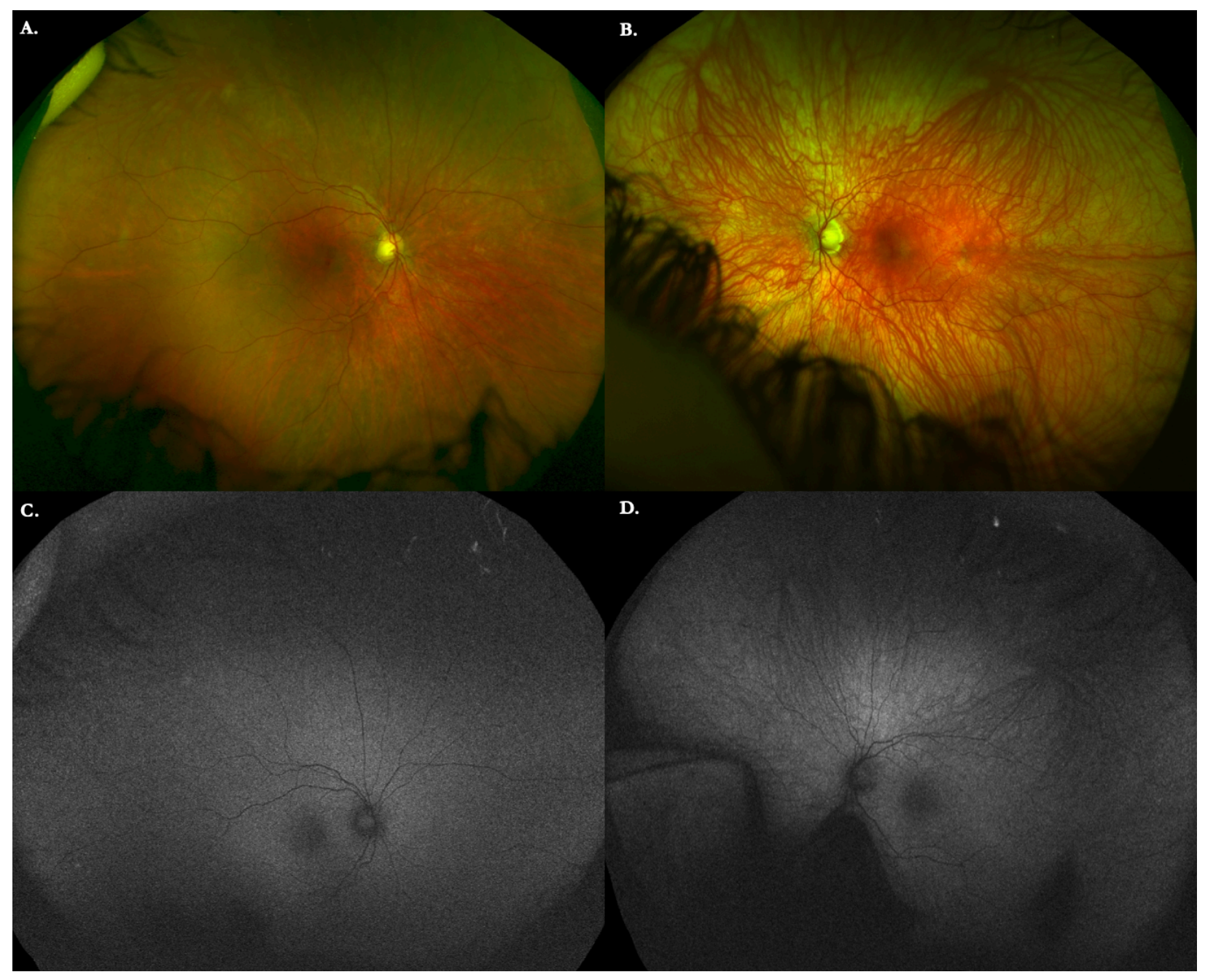

Figure 1 - Ultra-widefield color and autofluorescence images from the patient. Ultra-widefield color fundus photographs from the right (A) and left (B) eyes demonstrate mild nasal hypopigmentation in the right and diffuse choroidal hypopigmentation of the left fundus. Corresponding fundus autofluorescence from the right (C) and left (D) eyes demonstrate mild generalized hyperautofluorescence in the left fundus. 


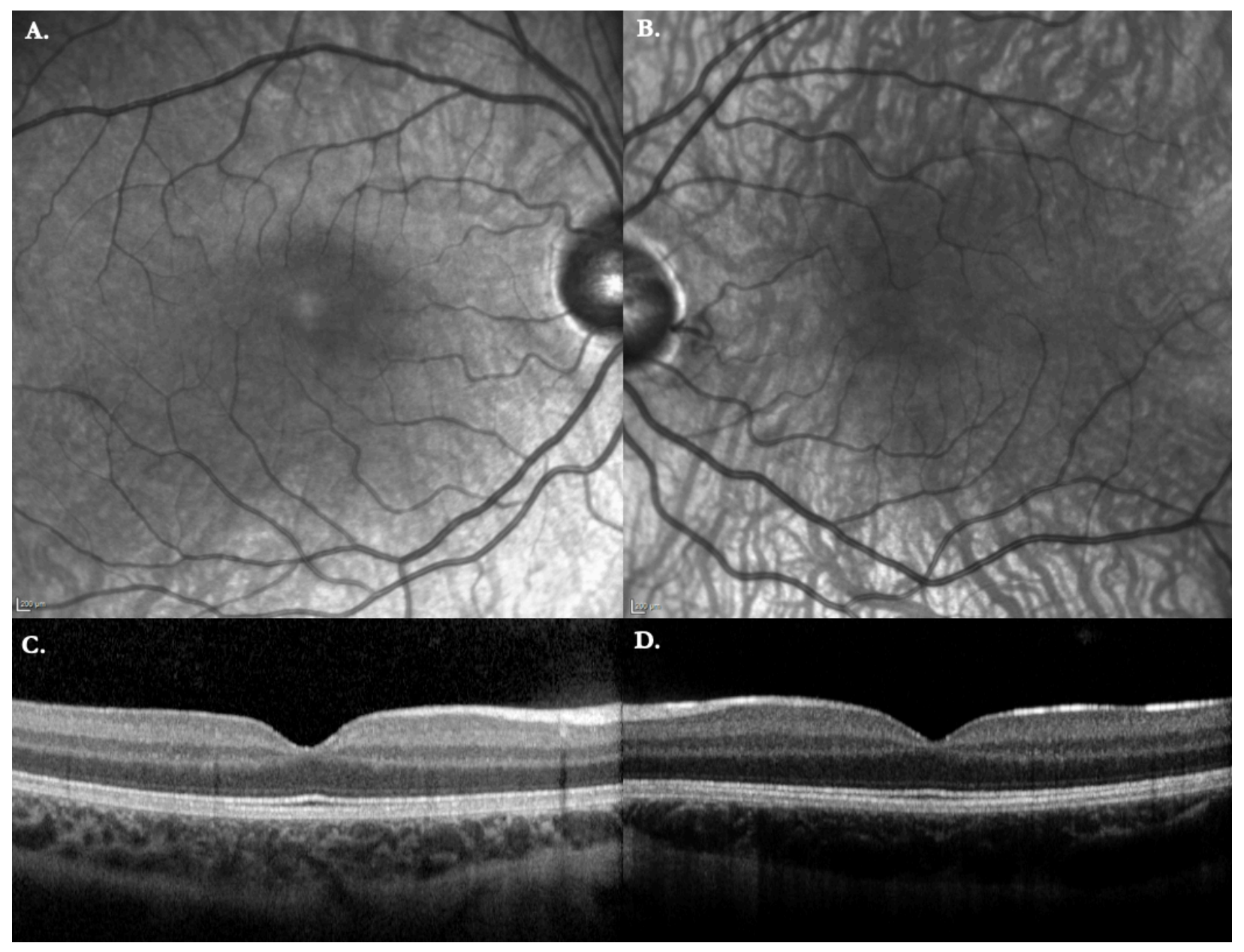

Figure 2-Optical coherence tomography images from the patient. The infrared reflectance image of the left eye (B) demonstrates more readily apparent choroidal vessels in comparison to the right eye $(\mathrm{A})$. The corresponding b-scans (C and $\mathrm{D})$ demonstrate normal macular architecture. 


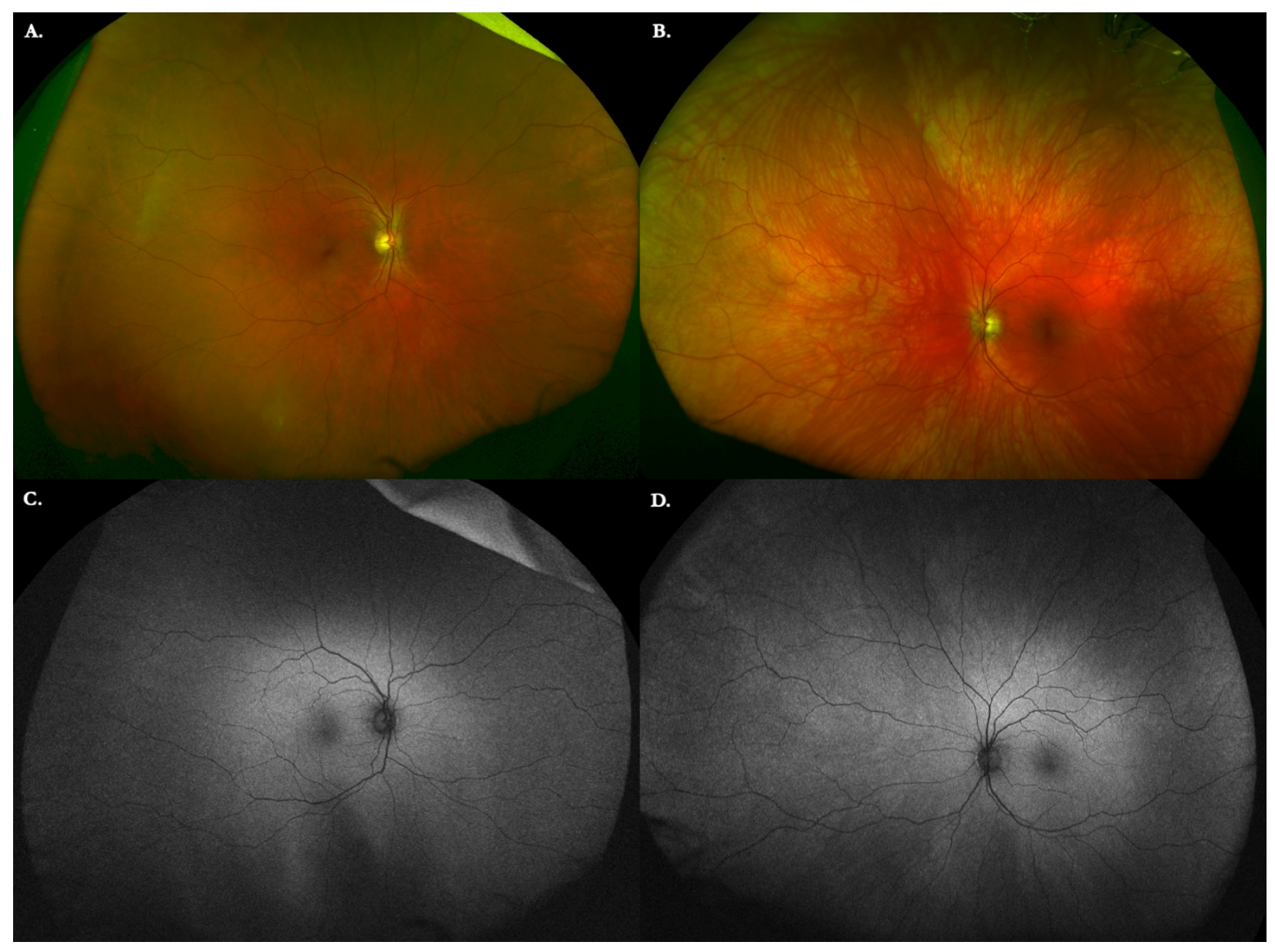

Figure 3 - Ultra-widefield color and autofluorescence images from the patient's mother. Ultra-widefield color photographs from the right (A) and left (B) eyes and corresponding fundus autofluorescence (C and D) from the mother demonstrate a similar pattern to the son. There is diffuse left choroidal hypopigmentation, with mild generalized hyperautofluorescence. Right eye imaging is normal. 